\title{
Application of soft sensor technology in HVAC system test
}

\author{
Changbao Guo ${ }^{1, *}$ \\ ${ }^{1}$ Dezhou Key Laboratory of High Efficiency Heat Pump Air-conditioning Equipment and System Energy-saving Technology, Shandong \\ Huayu Institute of Technology, Dezhou, Shandong
}

\begin{abstract}
With the rapid development of modern society, the measurement and control requirements of key variables of air conditioning test technology are also increasing. When the parameters of HVAC system are measured and controlled, there are a large number of sensors and instruments used. When sensor failure or measurement error distortion occurs, the air conditioning system cannot operate normally and automatically. At the same time, some variables in the test parameters cannot be directly collected by the sensor. In order to solve these problems, researchers have focused on soft measurement technology in recent years, which provides a good breakthrough for the measurement and control of air conditioning system test parameters. This paper expounds the application of soft measurement technology in HVAC system.
\end{abstract}

\section{Preface}

In HVAC system testing, it is often encountered that the controlled variables of key parameters cannot be measured online, or can be measured online. However, due to some sensor failures, the feedback data is inaccurate and difficult to control. For similar problems, the traditional solution is to control the factors that can better reflect and affect the controlled variables, in order to achieve the purpose of controlling the controlled variables. However, with the development of modern science and technology, the industrial process control system is becoming increasingly complex, and the required output variables are difficult to be detected by conventional detection techniques. Therefore, the idea of soft measurement is proposed [1].

As a development direction of indirect measurement, soft measurement technology is a research hotspot of modern detection. When some parameters cannot be directly collected by the sensor, the main output variables that are difficult to detect can be estimated according to the correlation between the relevant process variables in the system through the easily detected process auxiliary variables. Using soft measurement technology can not only reduce the instrument, but also bring great convenience to system installation and maintenance. At the same time, the soft measuring instrument has incomparable advantages in realization, universality, flexibility and cost. Therefore, it is of great significance to correctly understand the application of soft measurement technology in HVAC system testing.

\section{Soft-sensing technology}

\subsection{The basic principle of soft measurement technology}

Soft sensor technology should be applied to real time estimation, fault redundancy, intelligent correction and multiplexing in industrial process.It is the use of easy to measure the process variables (called auxiliary variables such as pressure, temperature, etc.) and the process variables to be measured (called the dominant variables, such as product distribution, material composition) between the mathematical relationship (soft measurement model), through a variety of mathematical calculation and estimation, using the form of software to realize the measurement of the process variables. Obviously, soft measurement is to construct a soft measurement model with easy-to-measure variables as independent variables and dominant variables as dependent variables, and obtain the value of dominant variables with computer software through various complex mathematical calculations and estimates [2]. At present, the main way to solve the problem is to use computer system to calculate the unmeasurable variables from the measured variables in the process. Figure 1 shows the relationship between the input and output of the soft sensor model used in the process control system and the input and output of the object. 


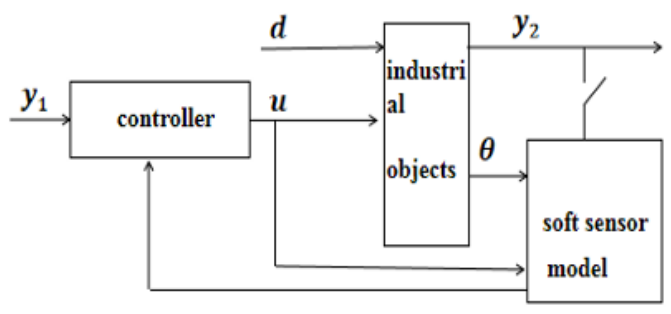

Fig. 1 Input and output of soft-sensing model in process ontrol.

\subsection{Establishment of mathematical model for soft measurement of air conditioning system}

The basic principle of soft measurement technology is based on the relationship between some process variables and other variables in the process, using computer technology, according to some easily measured auxiliary variables, calculate the dominant variable. The calculation is carried out according to the mathematical model between the auxiliary variable and the dominant variable [2]. The core of soft measurement technology is to establish the mathematical model of the object. The quality of the object mathematical model is directly related to the calculation results of the soft sensor. Soft sensor technology can be divided into mechanism modeling and experience modeling according to its modeling methods. The combination of mechanism modeling and empirical modeling can accommodate the advantages of both and complement the disadvantages of each. Modeling by combining mechanism with experience is a practical method, which is widely used at present.

Parameter detection is to achieve optimal control of the system. Therefore, the establishment of mathematical model of soft measurement technology in HVAC system should not only consider the main factors affecting the performance of soft measurement, but also determine to adopt reasonable and more realistic methods according to the requirements of control and the actual detection requirements of parameter changes.

\section{Application of soft sensor technology in HVAC system test}

\subsection{Application of soft sensor technology based on fuzzy control in HVAC system test}

\subsubsection{Fuzzy control of chiller}

Because the refrigerating capacity of refrigeration and air conditioning system is realized by adjusting the refrigerant circulation of chillers. The refrigerant circulation is usually controlled by electronic expansion valve, the purpose is to ensure that the refrigerant capacity and heat load match, so that the evaporator outlet refrigerant superheat stability in a certain range. However, the thermal inertia of the electronic expansion valve temperature sensing system is large, and the hysteresis phenomenon is serious, which leads to the poor performance of the control system, and even the phenomenon of unstable control and out of control. And the refrigeration system has the characteristics of large lag and nonlinear, there are many uncertain external factors, it is difficult to establish an accurate mathematical model. At present, the control field is still a large number of PID control mode: PID parameter selection and adjustment inconvenience is still a common problem. Because fuzzy control introduces human logic thinking mode, the fuzzy controller has certain adaptive control ability and strong robustness and stability, so it is especially suitable for the actual system without accurate mathematical model [3]. The schematic diagram of the fuzzy controller for the chiller is shown in Figure 2.

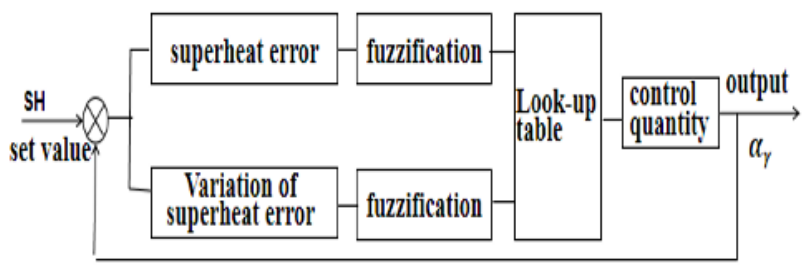

Fig. 2 Schematic diagram of fuzzy controller for chiller.

\subsubsection{Fuzzy control of terminal equipment}

Because the load of the air-conditioned room is always in change, an experienced operator can always control the temperature of the room in a relatively stable state through various methods. The operator obtains information from the instrument and the display, analyzes and judges according to the existing experience, and makes control decisions. This process does not go through accurate calculation and rigorous reasoning, is a fuzzy reasoning decision-making process. Fuzzy control is to imitate the thinking mode of human brain to control. Therefore, the soft sensor technology based on fuzzy control method can be used to adjust the room temperature. The fuzzy control system diagram is shown in Fig. 3.

fuzzy controller

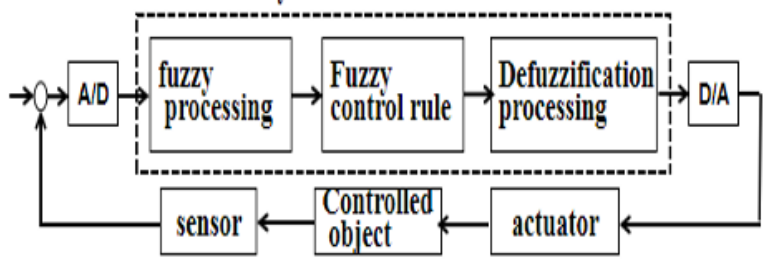

Fig. 3 Schematic diagram of fuzzy control system.

The indoor temperature is measured by the temperature sensor, and the digital quantity $\mathrm{T}$ corresponding to the measured temperature value is given by the input channel A / D conversion. The computer compares $\mathrm{T}$ with the set value S ( digital quantity), and obtains the digital quantity e of temperature deviation, and calculates the temperature change rate $\mathrm{e}$ '. e and e ' are used as the input of control variables. After fuzzification, the control outputs U1 and U2 are obtained by looking up the table, which corresponds to the increment of cooling capacity and air volume of the system at this time. The change of cooling capacity is realized by adjusting the 
cold water flow through the electric proportional control valve, and the fan air flow is realized by adjusting the fan speed by its voltage regulating circuit.

At present, in the HVAC system test, there are many detection variables in the air conditioning system. In order to control the system, data acquisition and processing is the basis. Without the corresponding sensor data acquisition, there is no strong evidence in the process of online control. The soft measurement technology based on fuzzy control can be used as the research direction of air conditioning system parameter information acquisition. It can not only reduce the number of instruments, but also avoid the setting of complex detection points, which brings great convenience to the installation and maintenance of the system. Therefore, the application research of fuzzy control technology in the field of central air conditioning has been deepened, and the control target parameters have developed from single temperature control to the control of comfort index. In terms of control strategy, it develops from simple fuzzy control based on query table method to intelligent fuzzy control.

\subsection{Application of soft sensor technology based on artificial neural network in HVAC system test}

Artificial neural network is a simplified model of human central nervous system. It is an operational processing system composed of many simple, highly interconnected processing units (called neurons) that process information in the form of dynamic responses to external input. Because artificial neural network has the functions of selflearning, associative memory, self-adaptation and nonlinear approximation, the soft sensor based on artificial neural network can directly establish the model according to the input and output data of the object without a priori knowledge of the object. This model has strong on-line correction ability and strong robustness, and has a good application effect for nonlinear and large lag systems.

The soft sensor method based on neural network mainly uses the powerful modeling ability of neural network to describe the relationship between auxiliary variables and dominant variables by using neural network instead of the conventional mathematical model, and completes the mapping from measurable information space to dominant variables. This kind of method is to take auxiliary variables as the input of artificial neural network and dominant variables as its output, and solve the soft measurement problem of unmeasurable variables through network learning. For example, Gibson has successfully developed an.ga (genetic algorithm) optimal energy manager for on-line operation of building central cooling system, which makes HVAC equipment run dynamically and optimally. Darrell D. Massie implemented ANN adaptive and predictive control strategies in the central HVAC system [4].

\subsection{Application of soft sensor technology in VAV air-conditioning system}

\subsubsection{Adopts soft sensor technology to automatically adjust VAV system}

In the HVAC system test equipment, VAV air conditioning box is the most critical equipment in the HVAC system, and its control principle is mostly the use of air volume sensor and temperature sensor signals through the regulator to the frequency converter, the frequency converter to adjust the VAV parameters. This control technology is difficult to achieve ideal results due to sensor fault or measurement error. So we adopt soft measurement technology, using computer software technology, the blower of the frequency converter control signal and the valve opening as auxiliary variables, the room air output as the dominant air volume, artificial neural network is adopted to establish the mathematical model of soft measurement between the dominant and auxiliary variables directly influences the precision of the mathematical model of soft measurement result.

Due to the change of blower inverter control signal to send air volume is larger, the influence of the soft measurement technology in alternative air flow sensor into actual operation, in order to further improve the performance of the variable air volume air conditioning system, using the technique of feedforward control, to offset from the blower inverter control signal changes affect delivery.Fig. 4 shows the neural network used for soft sensor.

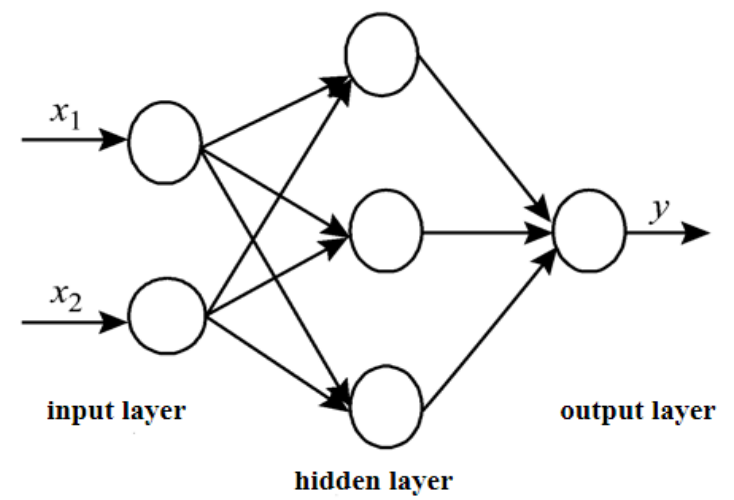

Fig. 4 Neural network for soft measurement.

\subsubsection{Dynamic detection of VAV air conditioning system is carried out by soft sensor technology}

The soft-sensing technology is used to monitor the operation of air volume sensor in the air conditioning box, and the actual measured value of the air volume sensor is compared with the results obtained by the soft measurement in the operation process of the variable air volume air conditioning. When the deviation between the two reaches or exceeds a certain degree (such as $10 \%$ ), it can be considered that the air volume sensor fails and an alarm signal is generated. At the same time, the soft measurement technology is used to replace the air volume sensor for actual operation, that is, in the case of air volume sensor failure, the variable air volume air 
conditioning system can still ensure good operation performance. As shown in Fig. 5.

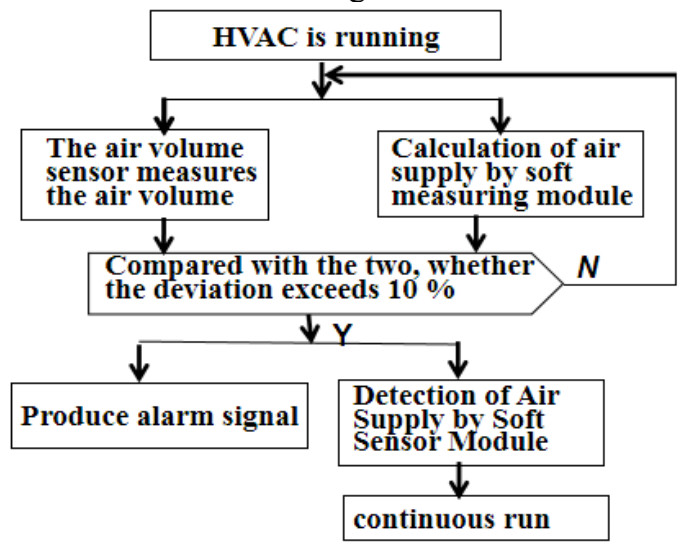

Fig. 5 The flow chart of soft measurement technology monitoring air volume sensor.

Because the change of the control signal of the fan inverter has a great influence on the air supply volume, when the soft sensor is used to replace the air volume sensor in the actual operation, we can use the feedforward control technology to further improve the operation performance of the VAV air conditioning system. This method can counteract the influence of the change of the control signal from the fan inverter on the air supply volume. Fig. 6 shows the schematic diagram of the air volume control loop with feedforward control.

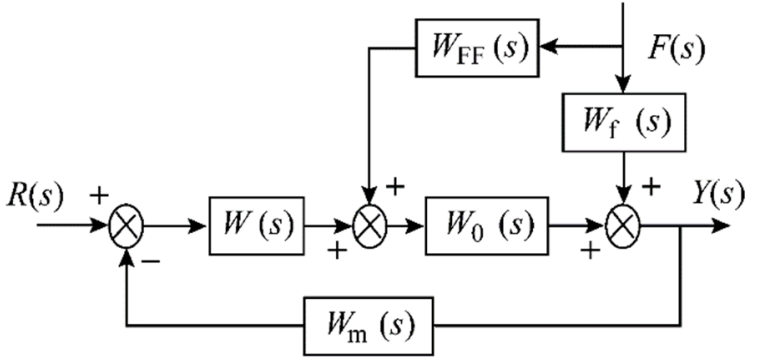

Fig. 6 Schematic diagram of air volume control circuit with feedforward control

\section{Conclusion}

With the progress of science and technology and the continuous improvement of people ' $s$ comfort requirements for $\mathrm{HVAC}$, the combination of measurement technology and control technology provides guarantee for the optimization control system of indoor environment. The application of advanced test technology represented by frequency conversion technology and fuzzy control technology in the field of HVAC has also attracted more and more attention. HVAC testing technology has gradually developed into the field of industrial process control technology. In recent years, the combination of artificial neural network technology with frequency conversion and fuzzy technology in soft measurement modeling has made rapid development in the field of HVAC testing technology.

\section{Acknowledgments}

This paper for the project "PBL teaching method in the teaching of building environment test technology, exploration and practice of" one of the achievements, focuses on the application of soft measurement technology in hvac system test, and the advanced test technology into the building environment test technology of actual case teaching, has been to improve the students' test skills. In the process of writing the paper, I got the technical support from the teachers of my project team and gave.

\section{References}

1. Yu, J.T. (2008) Soft sensor technology and its application [J]. AutomationInstrumentation, 29(1): 17.

2. Yu, J.J. (1996) Zhou Chunhui. Soft sensor technology in process control $[\mathrm{J}]$. Control Theory \& Applications, 13(3): 137-144.

3. Li, Y.S. (2007) Application of Intelligent Fuzzy Control Technology in Energy Saving Control of Central Air Conditioning [J]. Power Demand Side Management, 9(5): 77-78.

4. Shen, S.y. (2010) Neural Network System Theory and Its Application [M]. Beijing Science Press, The Beijing. 